\title{
XXXVI. Krystallmessungen.
}

\author{
Von \\ C. Hlawatsch in Wien.
}

(Mit 4 Textliguren.)

\section{Neue Messungen am Raspit von Brokenhill (N. S.-Wales).}

Die mineralogisch-petrographische $\Lambda$ bteilung des $\mathrm{k}$. k. naturhistorischen Hofmuseums in Wien erhielt im Jahre 1905 unter einem Geschenkposten aus Sydney auch ein Stück von Brokenhill, auf welchem in der bekannten Paragnese mit Stolzit und Psilomelan ca. $1 \mathrm{~mm}$ große, anscheinend gut ausgebildete Krystalle von Raspit saßen. Die Abteilungsleitung hatte die Liebenswürdigkeit, dem Verf. behufs Messung eine Anzahl Krystalle zur Verfügung zu stellen, wofür ihr an dieser Stelle der ergebenste Dank ausgesprochen sei. Leider erfüllten die Krystalle die auf sie gesetzte IIoffnung, die Elemente des Raspit von Brokenhill genauer als es bis jetzt der Fall war, bestimmen zu können, nicht. Die Reflexe waren nur an wenigen Krystallen gut, die Fläche $e\left\{\bar{T}_{01}\right\}$ fehlte durchgängig, während die Messungen für die von Hussak zuerst am Raspit von Sumidouro ${ }^{1}$ ) beobachtete Fläche $p\{122\}$, die an den hier besprochenen Krystallen die Fläche $d\{011\}$ an Ausdehnung übertraf, von den berechneten so sehr abweichende Werte ergaben, daß sie für die Bildung des Mittels nicht angewendet werden konnten. Die Beobuchtungen ergaben zudem, daß das Zonenstück zwischen $d$ und $p$ mitunter durch einen ununterbrochenen Reflexzug vertreten war; öfters trat neben $d$ die $\mathrm{zu}$ ihr vicinale $\delta\{1.12 .12\}$ auf, dieselbe sogar mitunter vertretend. Es war daher zu erwarten, daß auch $p$ nicht ganz in seiner richtigen Lage sich befinde; außerdem wird infolge des Index der Fehler bei der Berechnung von $p_{0}$ verdoppelt. Von sieben gemessenen Krystallen konnten nur vier zur Rechnung verwendet werden, $d$ trat haupt-

1) Centralbl. f. Min. usw. 1903, 723-72:. Rel. diese Zeitschr. 41, 647. 
sächlich an den weniger gut ausgebildeten Krystallen auf. In folgender Tabelle I sind die Resultate der Messung am Goldschmidtschen Goniometer bei Polarstellung nach der Orlhodomenzone zusammengestellt; in der letzten Columne ist das Mitlel aus sämtlichen zur Rechnung verwendeten Messungen am Raspit von Brokenhill, welche der Verf. durchgeführt hat ${ }^{1}$ ), beigefügt.

Tabelle I.

\begin{tabular}{|c|c|c|c|c|c|c|c|c|c|c|}
\hline$\stackrel{\breve{d}}{\Xi}$ & 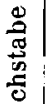 & & $\begin{array}{l}\text { I. } \\
\text { nbol }\end{array}$ & I & & $\begin{array}{c}\text { III. } \\
\text { Anz.der }\end{array}$ & Mit & chler & $\begin{array}{r}\mathrm{v} \\
\text { Mitte } \\
\text { Broke }\end{array}$ & für \\
\hline$z$ & 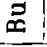 & Gdt. & Miller & $\varphi$ & 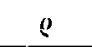 & Flächen & $\mathscr{F}$ & e & $\varphi$ & $\varrho$ \\
\hline 1 & $a$ & $\infty 0$ & 100 & $89^{0} 56^{\prime}$ & $90^{0} 0^{\prime}$ & 6 & $4 y$ & $0^{\prime}$ & $90^{\circ} 0^{\prime}$ & $90^{0} 0^{\prime}$ \\
\hline 2 & $b$ & $0 \infty$ & 010 & - & - & 一 & - & 一 & 一 & $\begin{array}{ll}0 & 0\end{array}$ \\
\hline $\mathbf{3}$ & $c$ & 0 & 001 & 1742 & $90 \quad 0$ & 12 & $5 !$ & 0 & 1741 & $90 \quad 0$ \\
\hline 4 & $m$ & $\infty$ & 110 & - & - & - & 二 & - & - & - \\
\hline 5 & $d$ & 01 & 011 & 1745 & 437 & 8 & 9 & 4 & $\begin{array}{lll}17 & 41\end{array}$ & 4316 \\
\hline 6 & $e$ & $\overline{10}$ & $\overline{1} 01$ & - & - & - & - & - & $\begin{array}{ll}-28 & 48\end{array}$ & $90 \quad 0$ \\
\hline 7 & $f$ & ${ }_{2}^{70} 0$ & $\overline{1} 02$ & -641 & $90 \quad 0$ & 2 & 48 & 0 & - & - \\
\hline 8 & $p$ & 1 & 122 & $36 \quad 11$ & 4754 & 2 & 4 & $2 !$ & - & - \\
\hline 9 & $\delta$ & 19 & 1.12 .12 & 2149 & 4355 & 4 & 13 & 4 & - & - \\
\hline
\end{tabular}

Aus diesen Messungen berechnet sich, wenn $p$ zur Bestimmung von $p_{0}$ verwendet wird:

$$
\begin{gathered}
p_{0}=0,7899, q_{0}=1,0664, \mu=72^{0} 17^{\prime} \\
a: b: c=1,4173: 1: 1,1195 ; \beta=107043^{\prime} .
\end{gathered}
$$

Die Elemente des Raspit von Brokenhill waren in der letzten diesbezüglichen Publication:

$$
p_{0}=0,8319, q_{0}=1,0587, \mu=72^{019^{\prime}} .
$$

Wie man sieht, ist hier eine sehr große Differenz in der Größe $p_{0}$. Aus den oben angeführten Gründen wurde daher $p$ von der Berechnung des Mittels ausgeschlossen und die in Col. $\mathrm{V}$ der Tabelle I angeführten Mittelzahlen aus sümtlichen bisherigen Beobachtungen des Verfs. am Raspit von Brokenhill zur Berechnung der Elemente dieses Raspitvorkommens verwendet. Danach berechnen sich:

$$
\begin{gathered}
p_{0}=0,8275, q_{0}=1,0624, \mu=72^{0} 19^{\prime}=180^{0}-\beta ; \\
a: b: o=1,3475: 1: 1,1151 .
\end{gathered}
$$

Der Raspit von Sumidouro hatte ergeben:

$$
\begin{gathered}
p_{0}=0,8286, q_{0}=1,0618, \quad \iota=72027^{\prime} ; \\
a: b: c=1,3440: 1: 1,1136 .
\end{gathered}
$$

Da die Differenzen der Elemente des Raspit beider Fundorte kleiner

1) Ann. d. k. k. naturh. Hofmus. 1897, 12, 39. Diese Zeitschr. 1899, 31, 9. 
sind als bei Brokenhill allein, so kann man aus beiden das Mittel nehmen, wobei natürlich für $e$ die Zahlen von Sumidouro mit dem doppelten Gewichte eingeführt werden müssen.

Die Winkel bei Polarstellung nach der Orthodomenzone sind dann:

\begin{tabular}{|c|c|c|}
\hline Fläche & $\varphi$ & $\varrho$ \\
\hline$a$ & $900 \quad 0^{\prime}$ & $9000^{\prime}$ \\
\hline$c$ & 1737 & $90 \quad 0$ \\
\hline$d$ & 1737 & 4316 \\
\hline e & -2854 & 90 \\
\hline
\end{tabular}

Nach diesen Werten wurden die Elemente und die Winkeltabelle für normale Aufstellung neu berechnet (s. Tabelle II). Zum Vergleich der gemessenen und der berechnelen Winkel sei darauf hingewiesen, daß $\varphi^{\prime}=\xi_{0}$, $\varrho^{\prime}=90^{\circ}-\eta$ ist, wenn $\varphi^{\prime}$ und $\varrho^{\prime}$ die Positionswinkel bei Polarstellung nach der Orthodomenzone bedeuten.

Tabelle II. Elemente.

\begin{tabular}{|c|c|c|c|c|c|}
\hline$a=1,34497$ & $\lg a=0,12871$ & $\lg a_{0}=0,08156$ & $\lg p_{0}=9,91844$ & $a_{0}=1,20658$ & $p_{0}=0,82878$ \\
\hline$c=1,11468$ & $\lg c=0,04715$ & $\lg b_{0}=9,95285$ & $\lg q_{0}=0,08629$ & $b_{0}=0,8$ & $q_{0}=$ \\
\hline$\stackrel{\mu=0}{1800-\beta}=72023^{\prime}$ & $\underset{\sin \mu}{=\lg h}=9,97914$ & $\frac{\lg e}{=\lg }=9,48094$ & $\lg \frac{p_{0}}{q_{0}}=0,89215$ & $h=0,95310$ & $e=0,30265$ \\
\hline
\end{tabular}

\begin{tabular}{|c|c|c|c|c|c|c|c|c|c|c|c|c|c|}
\hline $\begin{array}{l}\mathrm{g} \\
\mathrm{E} \\
\mathrm{g}\end{array}$ & 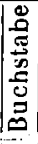 & $\begin{array}{l}\text { Sy } \\
\text { Grlt. }\end{array}$ & $\begin{array}{l}\text { mbole } \\
\text { Miller }\end{array}$ & $\varphi$ & 0 & $\xi_{0}$ & $r_{0}$ & $\xi$ & & $\eta$ & $\begin{array}{c}x^{\prime} \\
\text { (Prismen) } \\
x: y\end{array}$ & $y^{\prime}$ & $\begin{array}{l}d^{\prime}= \\
\operatorname{tg} \varrho\end{array}$ \\
\hline 1 & $a$ & $\infty$ & 100 & $90^{0} 0^{\prime}$ & $90^{\circ} 0^{\prime}$ & $90^{\circ} 0^{\prime}$ & $000^{\prime}$ & $90^{\circ} 0^{\prime}$ & & $00^{\prime}$ & $\infty$ & 0 & $\infty$ \\
\hline 2 & $b$ & $0 \infty$ & 010 & 00 & $90 \quad 0$ & 00 & $90 \quad 0$ & 00 & 90 & 0 & 0 & $\infty$ & $\infty$ \\
\hline 3 & $c$ & 0 & 001 & $90 \quad 0$ & 17.37 & 1737 & $\begin{array}{ll}0 & 0\end{array}$ & 1737 & 0 & 0 & $0,317 \dot{j} 4$ & 0 & 0,31754 \\
\hline 4 & $m$ & $\infty$ & 110 & $37 \ddot{57}$ & 190 & $90 \quad 0$ & 900 & $3757 \frac{1}{2}$ & 52 & $2 \frac{1}{2}$ & 0,78010 & $\infty$ & $\infty$ \\
\hline 5 & $d$ & 01 & 011 & 1554 & 4912,8 & 1737 & $48 \quad 6,2$ & 1158,3 & 46 & 44 & $0,3+754$ & 1,11462 & 1,15903 \\
\hline 6 & $e$ & TO & $\overline{101}$ & $\begin{array}{ll}-90 & 0\end{array}$ & 2854 & -2854 & 00 & -2854 & 0 & 0 & $-0,55202$ & 0 & 0,55202 \\
\hline 7 & $f$ & 70 & T02 & -900 & 644 & -644. & 00 & -644 & 0 & 0 & $-0,11724$ & $\infty$ & 0,11724 \\
\hline 8 & $p$ & $\frac{1}{2} 1$ & 122 & $\begin{array}{ll}34 & 1\end{array}$ & 8321,9 & 36 ธั7,3 & $48 \quad 6,2$ & 2640,4 & 41 & 41,6 & 0,71703 & $\overline{1}, 11462$ & 1,34481 \\
\hline 9 & $\delta$ & $T^{\frac{2}{1}}-1$ & 1.12 .12 & 1917 & 494,6 & 2118,3 & $48 \quad 6,2$ & 1433,6 & 46 & 4,9 & 0,3900 & 1,11462 & 1,18091 \\
\hline
\end{tabular}

Hier, wie in allen folgenden Tabellen, sind die fünften Decimalen bei den linearen Werten und die Bruchteile der Minuten bei den Winkeln nur zur Controle der Rechnung angegeben, mit der Genauigkeit der Messung haben dieselben gar keinen Zusammenhang.

Die bei den früheren Krystallen von Brokenhill stets vorhandene Zwillingsbildung nach (100) war auch auf der hier beschriebenen Stufe häufig, einfache Krystalle waren jedoch nicht selten; die Zwillinge waren 
teils als Juxtapositions-, teils als Penetrations-Zwillinge entwickelt. Infolge eines Unwohlseins des Verfs. war es ihm leider nicht mehr möglich, eine Zeichnung der Krystalle anzufertigen, für die Bestimmung der Symbole von $d$ auf graphischem Wege ist Derselbe IIerrn Custos Dr. Köchli in zu großem Danke verpflichtet.

\section{Eine trikline, rhodonitähnliche Schlacke.}

Herr Prof. v. Groth hatte die Liebenswürdigkeit, dem Verf. gelegentlich seines $\Lambda u$ fenthaltes in München im Jahre 1903 einige Schlacken zur Untersuchung zu übergeben, darunter auch eine Schlacke unbekannter Provenienz und eine dieser ähnliche vom Bleihochofen „Germaniasmelter " bei Salt Lake City. Es sei dem Autor hier gestattet, Herrn Prof. v. Groth hiermit den aufrichtigsten Dank für die freundliche Überlassung des Materiales auszusprechen. Die erstere Schlacke führte in den Höhlungen zahlreiche dünntafelige, bald unregelmäßig sechsseitige, bald nach der als Prismenzone gewählten Zone der Spaltflächen gestreckte Krystalle mit schief abgeschrägten Enden. Schon die erste Untersuchung an kleinen Spaltblättchen sowie einige optische Beobachtungen zeigten die Zugehörigkeit zum triklinen Systeme; es wurden Winkel zwischen den Spaltfiächen und der Tafelebene von $95^{\circ} 30^{\prime}, 69^{\circ} 39^{\prime}$ und $44^{\circ} 8^{\prime}$ erhalten. Durch die Tafelebene sieht man im convergenten Lichte das Bild einer optischen Axe, vom Rande des Gesichtsfeldes mehr oder weniger entfernt, deren Balken in jeder Stellung ziemlich gerade gestreckt ist. In der Mehrzahl der Fülle liegt die spitze, positive Bisectrix der Normalen auf die Tafelebene näher, die optische Axe bildet mit der letzteren einen Winkel von ca. 150. Dispersion ziemlicli deutlich, $\varrho>v$. Mitunter aber finden sich Blättchen, bei denen die optische Axe weiter von der Normalen entfernt ist, dann ist die positive Bisectrix zweite Mittellinie. Der $\Lambda$ xenwinkel $2 \mathrm{~V}$ schwankt also um $90^{\circ}$.

Die Axenebene steht nahezu senkrecht auf der Blättchenebene, $\beta$ liegt im stumpfen Winkel, welchen die Tracen von (011) und (120) auf ihr bilden, der Winkel gegen die Prismenkante betrïgt $9^{0}-14^{0}$. Die Doppelbrechung scheint ziemlich stark $\mathrm{zu}$ sein, da man durch die etwa $0,2 \mathrm{~mm}$ dicken Blättchen mehr als einen ling um die Axe erblickt.

Die Farbe der Blättchen ist in reinem Zustande gelblichweiß, in der Regel aber sind dieselben von massenhaft eingeschlossenem Magnetitstaub schwarz gefärbt. Diese Einschlüsse machten eine quantitative Analyse unmöglich, war es doch sogar schon schwierig für die Bestimmung des specifischen Gewichtes mittels Rohrbachscher Lösung ein genügend reines Blättchen zu finden. Eine qualitative Probe ergab die Anwesenheit beträchtlicher Mengen von Mangan. Die Dichte war 3,44. Nach diesen Eigenschaften wäre zu erwarten gewesen, daß eine dem Eisenrhodonit 
Weibulls1) nahestehende Substanz vorliege, wobei (010) die Tafelebene wäre. Allein die krystallographische Untersuchung am Goldschmidtschen Goniometer ergab trotz der meist schlechten Reflexe aller Flächen mit Ausnahme der Tafelflüche eine beträchtliche Abweichung von den Winkeln des Phodonites und des Babingtonites in der Prismenzone; die Kopfflüchen zeigten gar keine Übereinstimmung mit einfachen Flächen dieser Gruppe.

Die Resultate der Messungen an zehn Krystallen, wobei oft das verkleinerte Fernrohr angewandt werden mußte, sind in 'Tabelle III zusammengestellt. Dabei ist die Ebene der Blättchen als $(100)$, die beiden Endflächen als $\{011\}$ und $\{0 T 1\}$ aufgefaßt.

Tabelle III.

\begin{tabular}{|c|c|c|c|c|c|c|c|c|c|c|c|}
\hline$\stackrel{\Xi}{\Xi}$ & 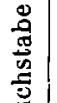 & Syn & bol & & & $\begin{array}{l}\text { Anz. der } \\
\text { Flächen }\end{array}$ & Mittl & ehler & $\begin{array}{r}\text { Zur Re } \\
\text { verwende }\end{array}$ & teW & $\begin{array}{l}\text { ing } \\
\text { Verte }\end{array}$ \\
\hline $\bar{z}$ & 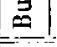 & Gdt. & Miller & $\varphi$ & $\varrho$ & & $\varphi$ & $\varrho$ & $\varphi$ & & $\underline{\rho}$ \\
\hline 1 & $a$ & $\infty 0$ & 100 & $95030^{\prime}$ & $90^{0} 1^{\prime}$ & 19 & $\mathbf{3}^{\prime}$ & $8^{\prime}$ & $95030^{\prime}$ & $90^{\circ}$ & $0^{\prime}$ \\
\hline 2 & $b$ i & $0 \infty$ & 010 & $0 \quad 0$ & 905 & 19 & $12 \%$ & 7 & 00 & 90 & 0 \\
\hline 6 & $M$ & $\infty \bar{\infty}$ & $1 \overline{1} 0$ & 13956 & 90 & 10 & 11 & 4 & 13952 & 90 & 0 \\
\hline 4 & $m$ & $\infty$ & 110 & 4510 & 90 & 2 & 4 & 0 & $45 \quad 112 !$ & 90 & 0 \\
\hline 5 & $t$ & $\infty 2$ & 120 & 2353 & 90 & 4 & 16 & 4 & $2538 \frac{1}{2}$ & 90 & 0 \\
\hline 7 & $d$ & 01 & 011 & 2645 & 28281 & $\ddot{5}$ & 40 & 11 & $2631^{2}$ & 28 & $25: \frac{1}{2}$ \\
\hline 8 & $e$ & $0 \overline{1}$ & $0 \overline{11}$ & $156 \quad 46$ & $\begin{array}{lll}31 & 131 \\
1\end{array}$ & 5 & $\left.52^{3}\right)$ & 3 & 15633 & 31 & 16 \\
\hline
\end{tabular}

Aus diesen Winkeln wurden die Elemente nach den Formeln:

$$
x_{1}=x_{0}+p_{1} p_{0} \sin r ; \quad x_{2}=x_{0}+p_{2} p_{0} \sin r,
$$

$$
\begin{gathered}
y_{1}=y_{0}+q_{1} q_{0}+p_{1} p_{0} \cos r, y_{2}=y_{0}+q_{2} q_{0}+p_{2} p_{0} \cos r, y_{0}=\frac{y_{1}+y_{2}}{2}, \\
p p_{0}=-\sin \alpha \\
q q_{0}
\end{gathered}
$$

berechnet. Dabei wurde $x_{1}$ mit $x_{2}$ ausgeglichen, und $\underset{q_{0}}{p_{0}}$ aus $\{1 \bar{T} 0\}$ und $\{120\}$ berechnet und so ausgeglichen, daß $\frac{q}{\{120\}}$ mit $\frac{1}{4}$ Gewicht eingeführt wurde. Auf diese Weise entstanden die in Tab. III, Col. IV angeführten Werte. In die Winkeltabelle wurde der Vollstïndigkeit halber auch die nicht beobachtete Fläche $c=\{001\}$ aufgenommen.

1) Diese Zeitschr. 1885, 10, 514.

2) Berechnet, nicht zur Rechnung verwendet.

3) Eine Messung mit einer Differenz von $2^{0} 27^{\prime}$ wurde mit $\frac{1}{8}$ Gewicht angerechnet; daher im miltleren Fehler die große Zahl. 
Tabelle IV. Elemente.

\begin{tabular}{|l|l|l|l|l|l|}
\hline$a=0,51826$ & $\alpha=93^{2} 6^{\prime} 30^{\prime \prime}$ & $p_{0}=0,46629$ & $\lambda=87058^{\prime} 33^{\prime \prime}$ & $x_{0}=0,23473$ & $d=0,23737$ \\
\hline$b=1$ & $\beta=1035912$ & $q_{0}=0,50581$ & $\mu=761717$ & $y_{0}=0,03532$ & $\delta=98033^{\prime} 26^{\prime \prime}$ \\
\hline$c=1,11588$ & $\gamma=8350$ & 8 & $r_{0}=1$ & $\nu=95$ 30 & $\begin{array}{c}h=0,97140 \\
=\cos \varrho_{0}\end{array}$ \\
\hline
\end{tabular}

Winkeltabelle.

\begin{tabular}{|c|c|c|c|c|c|c|c|c|c|c|c|c|c|c|c|c|}
\hline 这 & 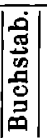 & $\begin{array}{l}\text { Sym } \\
\text { Gdt. }\end{array}$ & $\begin{array}{l}\text { bol } \\
\text { Mill }\end{array}$ & $\varphi$ & & $\varrho$ & & $\xi_{0}$ & $\eta_{0}$ & 10 & $g$ & $\boldsymbol{\xi}$ & $\eta$ & $x^{\prime}$ & $y^{\prime}$ & $\begin{array}{c}d^{\prime}= \\
\operatorname{tg} \varrho\end{array}$ \\
\hline 1 & $a$ & $\infty 0$ & 100 & $95030^{\prime}$ & 900 & $0^{\prime}$ & $90^{0}$ & $0^{\prime}$ & 900 & & 840 & $30^{\prime}$ & $-5030^{\prime}$ & $\overline{1} \overline{0}, 3850$ & - & $\infty$ \\
\hline 2 & $b$ & 000 & 010 & 00 & 0 & 0 & 0 & 0 & 90 & & 0 & 0 & $90 \quad 0$ & 0 & - & $\infty$ \\
\hline 3 & $c$ & o & 001 & 9833,5 & 13 & 43,9 & 13 & 35,1 & -2 & $\mathbf{5}$ & 13 & 34,5 & $-2 \quad 1,5$ & 0,24164 & $-0,03636$ & 0,24436 \\
\hline 4 & $m$ & $\infty$ & 110 & 4511,3 & 90 & 0 & 90 & 0 & 90 & 0 & 45 & 11,3 & $44 \quad 48,7$ & 1,00656 & - & $\infty$ \\
\hline $\mathbf{5}$ & $t$ & $\infty 2$ & 120 & 2538,5 & 90 & 0 & 90 & 0 & 90 & 0 & 25 & 38,5 & 6424,5 & 0,48003 & - & $\infty$ \\
\hline 6 & $M$ & $\infty \bar{\infty}$ & $1 \overline{1} 0$ & 13951,9 & 90 & 0 & 90 & 0 & 90 & 0 & -40 & 8,1 & 4951,9 & $-1,18605$ & - & $\infty$ \\
\hline 7 & $d$ & 01 & 011 & 2630,9 & 28 & 25,5 & 13 & 35,1 & 125 & $\mathbf{5 0 , 6}$ & 12 & 26,2 & $25 \quad 12,7$ & 0,24164 & 0,48436 & 0,54128 \\
\hline 8 & $e$ & $0 \overline{1}$ & $0 \overline{T 1}$ & 15633 & 31 & 16 & 13 & 35,1 & -29 & 7,2 & 11 & 55,2 & $\mid \begin{array}{ll}-28 & 26,1\end{array}$ & 0,24164 & $-0,55706$ & 0,60721 \\
\hline
\end{tabular}

Endlich sei noch eine Tabelle $V$ mit einigen der wichtigeren berechneten Kantenwinkeln und am Schlusse derselben zwei ebene Winkel auf (100) angereiht.

Tabelle V. Kantenwinkel.

\begin{tabular}{|c|c|c|c|c|c|}
\hline Buchstabe & Symbol & $\Delta$ & Buchstabe & Symbol & $\not$ \\
\hline $\begin{aligned} a & : m \\
a & : t \\
a & : M \\
m & : M \\
M & : t^{\prime} \\
b & : d \\
b & : e \\
a & : d \\
a & : e\end{aligned}$ & $\begin{array}{l}100: 110 \\
100: 120 \\
100: 1 \overline{10} \\
110: 1 \overline{1} 0 \\
1 \overline{1} 0: \overline{1} 0 \\
010: 011 \\
010: 0 \overline{11} \\
100: 011 \\
100: 0 \overline{1} 1\end{array}$ & $\begin{array}{lc}50018,7 \\
69 & 51,5 \\
44 & 24,9 \\
94 & 40,6 \\
63 & 46,6 \\
64 & 47,3 \\
61 & 33,9 \\
81 & 10,2 \\
75 & 26,1\end{array}$ & $\begin{array}{c}M^{\prime}: d \\
M: e \\
m: d \\
m: e \\
t: d \\
t^{\prime}: e \\
a m: a d \\
a M: a e\end{array}$ & 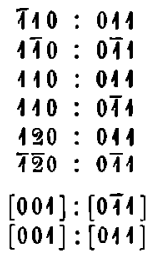 & $\begin{array}{lr}790 & 7,5^{\prime} \\
60 & 11,2 \\
63 & 11,5 \\
79 & 6,1 \\
61 & 34,6 \\
70 & 7,8 \\
63 & 11,6 \\
62 & 1\end{array}$ \\
\hline
\end{tabular}

Die Flächen $b, M, t$ sind Spaltflächen, von $m$ ist dies unsicher, da es zu selten beobachtet wurde. $M, m$ und $t$ sind stets so schmal, daß auch bei Anwendung des signalverkleinernden Fernrohres mit der Lupe die Flächenbeschaffenheit nicht erkannt werden kann. $b$ und $d$ weisen hüufig kastenförmige Vertiefungen auf, die nur einen schmalen Saum der Fläche übrig lassen.

Zwillinge scheinen nicht selten aufzutreten, doch gelang es nicht, das Gesetz zu ermitteln, da gerade an diesen Kopffächen fehlten. Es dürfte aber sowohl das Gesetz: Zwillingsebcne ist 010 - als auch: Zwillingsaxe ist $b$ - vorkommen, di einerseits Krystalle mit gemeinsamer $a$-Fläche aber 
unsymmetrischer Auslöschung, andererseits deutliche Knickungen auf der $a$-Flïche auftreten. Fig. 1 gibt ein idealisiertes Bild der sechsseitigen Krystalle, Fig. 2 die stereographische Projection. Auf ersterer sind sämtliche Flïchen zur Darstellung gebracht. Die punktierten Iinien sollen die Auslüschungsrichtungen auf $(100)$ darstellen.

Fig. 1.

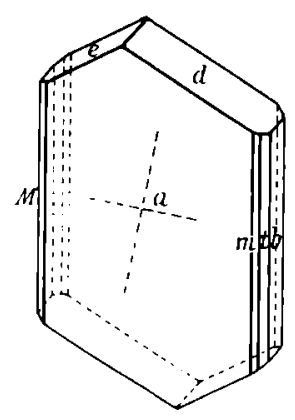

Fig. 2.

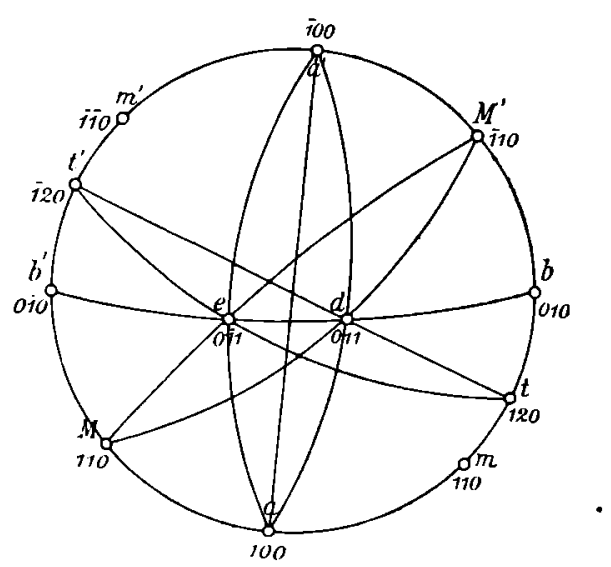

Die Schlacke vom Germaniasmelter erwies sich bei den Untersuchungen als mit der vorigen identisch; nur waren die Krystalle nach der Prismenzone nadelfürmig gestreckt, genauere Messungen lionnten daran nicht vorgenommen werden.

Da diesc Schlacke, wie aus den Spaltverhältnissen hervorgeht, und wie auch die Winkel der Prismenzone und der Kopflliichen trotz schlechter Reflexe zeigen, sich mit Rhodonit odcr Babingtonit nicht identificieren lïßt, wenn auch in der Prismenzone eine gewisse Analogie nicht zu verkennen ist; da ferner ihre Dichte der der Pyroxengruppe nahesteht, so vermutet der Verf., daß sie vielleicht ein Glied der von J. H. L. Vogt erkannten triklinen Modification der Verbindung ( $\mathrm{Mg}, \mathrm{Fe}, \mathrm{Mn}) \mathrm{SiO}_{3}$ darstellt. Er möchle daher vorschlagen, falls diese Schlacke nicht nachtrüglich mit einer schon gemessenen und bekannten Form identificiert wird, sie zu Ehren des Mannes, der vom Schlackenstudium ausgehend durch Anwendung der Sätze der physikalischen Chemie so bedeutende Fortschritte in Mineralogie und Petrographic erzielte, nach Hrn. Prof. Dr. Joh. H. L. Vogt in Kristiania, "Vogtit \& zu nennen; da ihm ja, falls des Verfs. Vermutung zutrillt, die Entdeckung dieser Substanz zuzuschreiben ist. 


\section{Dibromoxydesoxylbenzoin $\mathrm{C}_{6} \mathrm{H}_{5} \mathrm{CH}_{2} \mathrm{COC}_{6} \mathrm{H}_{2} \mathrm{Br}_{2} \mathrm{OH}+1$ aq.}

Die Krystalle dieser Substanz wurden von Herrn Ing. Fritz Glaßner in Bielitz dargestellt und dem Verf. in freundlichster Weise zur Messung überlassen, wofür Herrn Glaßner, der über die Darstellung in den Sitzungsberichten der $\mathbf{k}$. Akademie der Wissenschaften genauer berichten wird, der beste Dank ausgesprochen sei.

Die Krystalle sind 1-2 $\mathrm{mm}$ lang, nach der Prismenzone gestreckt, farblos. System: Monoklin. Klasse: prismatisch?.

Ausgebildet sind die Flächen $b\{010\}, a\{100\}, m\{110\}, o\{111\}$. Bald wiegen die Prismenllächen über die Pinakoide, bald $\{010\}$ über die Prismen vor, $\{100\}$ ist meist schmal. Ätzversuche mit Alkohol ergaben nur strichförmige Ätzfiguren, so daß die definitive Entscheidung, zu welcher Symmetrieklasse die Substanz gehürt, noch aussteht; wahrscheinlich ist es die prismatische. Die optischen Eigenschaften sind schwer zu untersuchen, da eine vollkommene Spaltbarkeit fehlt, nach $b$ scheint nur eine ziemlich deutliche zu gehen. An einem zufällig erhaltenen Präparate nach dieser Flïche ließ sich erkennen, daß $\gamma$ mit der Verticalaxe im spitzen Winkel [101]: [001] einen Winkel von ca. 410 bildet. Gemessen und zur Rechnung verwendet wurden sechs Krystalle, die Resultate sind in Tabelle VI zusammengestellt. $c\{001\}$ und $q\{011\}$ wurden nicht als Flächen beobachtet, an ihre Stelle trat an manchen Krystallen eine Abrundung der spitzen Ecke $o, o^{\prime}, a$; unter dem Mikroskope fand sich auch mitunter eine gerade, sehr kleine $\Lambda$ bstumpfung derselben vor. In Fig. 3 sind dieselben darum schraffiert.

Tabelle VI.

\begin{tabular}{|c|c|c|c|c|c|c|c|c|c|c|}
\hline $\begin{array}{l}\bar{\Phi} \\
\mathrm{E}\end{array}$ & $\begin{array}{l}\dot{3} \\
\stackrel{5}{0} \\
0\end{array}$ & & bol & & & Anz, der & & ehler & $\begin{array}{l}\text { Zur Re } \\
\text { verw }\end{array}$ & $\begin{array}{l}\text { bnung } \\
\text { ndet }\end{array}$ \\
\hline $\bar{z}$ & 吾 & Gdt. & Miller & $\varphi$ & 0 & Flächen & $\varphi$ & $\varrho$ & $\varphi$ & $e$ \\
\hline 1 & $b$ & $0 \infty$ & 010 & $001^{\prime}$ & $9002^{\prime}$ & 12 & $6^{\prime}$ & $3 y^{\prime \prime}$ & $\begin{array}{ll}0 & 0^{\prime}\end{array}$ & 9000 \\
\hline 2 & $a$ & $\infty 0$ & 100 & $\begin{array}{ll}89 & 59\end{array}$ & $90 \quad 0$ & 10 & $3 \frac{1}{2}$ & $0 \frac{1}{2}$ & $90 \quad 0$ & $90 \quad 0$ \\
\hline 3 & $c$ & 0 & 001 & - & - & - & $=$ & - & - & - \\
\hline 4 & $m$ & $\infty$ & 110 & 311 & 900 & 24 & $8 \frac{1}{2}$ & $3 !$. & 311 & 900 \\
\hline 5 & $q$ & 01 & 011 & -- & - & - & - & - & - & - \\
\hline 6 & $o$ & $\overline{11}$ & $\bar{T} 11$ & $\mid-26 \quad 30$ & $56 \quad 47$ & 12 & 4 & $3 \frac{4}{2}$ & -26 50 & $\begin{array}{ll}56 & 47\end{array}$ \\
\hline
\end{tabular}

Aus diesen Messungen berechnen sich die Elemente und die Winkeltabelle.

Tabelle VII. Elemente.

\begin{tabular}{|c|c|c|c|c|}
\hline$a=1,67719$ & $\lg a=0,22458 \lg a_{0}=0,09017$ & $\lg p_{0}=9,90983$ & $\left|a_{0}=1,23074\right|$ & $p_{0}=0,81251$ \\
\hline$c=1,36272$ & $\lg c=0,13441 \mid \lg b_{0}=9,86559$ & $\lg q_{0}=0,13077$ & $b_{0}=0,73382$ & $q_{0}=1,35134$ \\
\hline${ }_{-\beta}^{\mu}=82035^{\prime} 40^{\prime \prime}$ & $\left|\begin{array}{l}\lg h= \\
\lg \sin u=9,99636\end{array}\right| \begin{array}{l}\lg e= \\
\lg \cos u=9,11024\end{array} \mid$ & $\lg \frac{p_{0}}{q_{0}}=9,77906$ & $h=9,99165$ & $c=0,12890$ \\
\hline
\end{tabular}


Winkeltabelle.

\begin{tabular}{|c|c|c|c|c|c|c|c|c|c|c|c|c|}
\hline 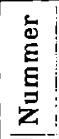 & $\begin{array}{l}0 \\
\frac{\pi}{0} \\
0 \\
0 \\
0 \\
0\end{array}$ & $\left|\begin{array}{l}\text { Syn } \\
\text { تु }\end{array}\right|$ & $\begin{array}{l}\mathrm{mb} . \\
\dot{\bar{B}}\end{array}$ & $\boldsymbol{\varphi}$ & $\varrho$ & $\xi_{0}$ & $\eta_{0}$ & $\xi$ & $\eta$ & $\begin{array}{c}x^{\prime} \\
\text { (Prismen } \\
x: y)\end{array}$ & $y^{\prime}$ & $\begin{array}{c}d^{\prime}= \\
\operatorname{tg} \varrho\end{array}$ \\
\hline 1 & $b$ & $0 \infty \infty^{\prime}$ & 010 & $000^{\prime}$ & $90^{0} 0^{\prime}$ & $000^{\prime}$ & $9000^{\prime}$ & $000^{\prime}$ & $9000^{\prime}$ & 0 & $\infty$ & $\infty$ \\
\hline 2 & $a$ & $\infty$ & & $90 \quad 0$ & $90 \quad 0$ & 900 & 00 & 900 & $\begin{array}{ll}0 & 0\end{array}$ & 0 & 0 & $\infty$ \\
\hline$\left.(3)^{1}\right)$ & $c$ & & 001 & & $7^{0} Z 4^{\prime} 20^{\prime \prime}$ & $7024^{\prime} 20^{\prime}$ & $\begin{array}{ll}0 & 0\end{array}$ & $7094^{\prime} 20^{\prime \prime}$ & $\begin{array}{ll}0 & 0\end{array}$ & 0,12998 & 0 & 0,12998 \\
\hline 4 & $m$ & $\infty$ & 1110 & 311 & $90^{0} 0^{\prime}$ & $9000^{\prime}$ & $90 \quad 0$ & $3101^{\prime}$ & 5859 & 0,60 & 一 & $\infty$ \\
\hline (5) & $q$ & 01 & {$[011$} & 527 & 3351 & $70 \geq 4^{\prime} 20^{\prime}$ & 5343,7 & 423,9 & 5ั3 39,9 & 90,12998 & 1,36272 & 1,36893 \\
\hline 6 & \begin{tabular}{|l|}
1 \\
0
\end{tabular} & $\overline{1} 1$. & $\pi 11$ & -2650 & 5647 & $-34^{0} 94,8$ & 5343,7 & $-2211,2$ & 4817 & & 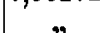 & 1,52717 \\
\hline (7) & $p$ & 11 & 111 & 3451,8 & 5836,8 & 4330,7 & 5343,7 & 2919,3 & 443 & 80,94934 & $\eta$ & 1,66080 \\
\hline
\end{tabular}

Tabelle VIII. Berechnete Kantenwinkel.

\begin{tabular}{|c|c|c|}
\hline Buchstabe & Symbole & 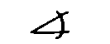 \\
\hline$b: o$ & $040: \overline{1} 11$ & 41042,7 \\
\hline$o: o^{\prime}$ & $\overline{1} 11: \overline{1} 11$ & $\begin{array}{ll}96 & 34,6\end{array}$ \\
\hline $0: a^{\prime}$ & $\overline{1} 11: \overline{100}$ & $64 \quad 48,8$ \\
\hline$o: m$ & $\bar{T} 11: 100$ & $\begin{array}{ll}63 & 33,9\end{array}$ \\
\hline$o: m^{\prime \prime \prime}$ & $\overline{111}: \overline{110}$ & $33 \quad 27$ \\
\hline
\end{tabular}

Fig. 3.

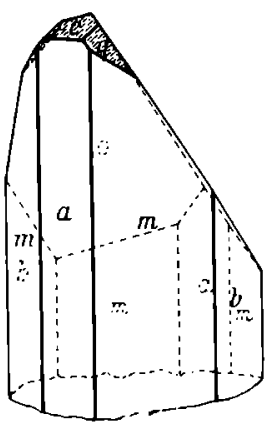

Fig. 4.

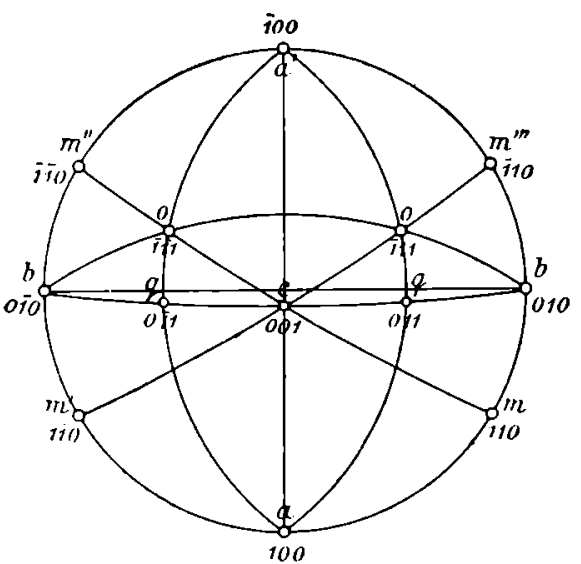

Fig. 3 gibt ein idealisiertes Bild jener Krystalle, bei welchen (110) nahe im Gleichgewichte mit (010) ausgebildet ist; wie bereits erwähnt, sind dabei die Flächen $c$ und $q$ schraffiert eingezeichnet, um die Abrundung der Ecke anzudeuten. Auch auf Fig. 4, welche die stereographische Projection darstellt, wurden diese Flächen eingetragen.

Wien, Juli 1906.

1) Die mit eingeklammerten Nummern bezeichneten Flächen sind nicht wirklich beobachtet, sondern nur der Vollständigkeit halber eingereiht. 